\title{
G

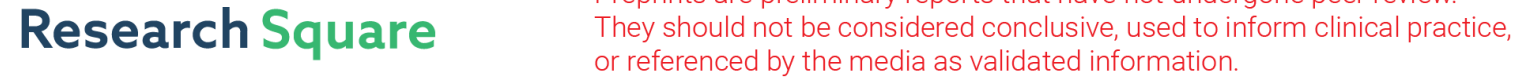 \\ Clinical analysis of severe acute respiratory syndrome coronavirus 2 (SARS-CoV-2) infection in children
}

\section{Guilang Zheng}

Department of Pediatrics, Guangdong Provincial People's Hospital (GDPH)囚Guangdong Academy of Medical Sciences $₫$ Guangzhou, China.

\section{Chuxing Xie}

Pediatric respiratory department, Dongguan Children's Hospital , Dongguan, China.

\section{Dongli Liu}

\author{
GDPH
}

\section{Guojing Ye}

Department of Pediatrics, Huizhou Third People's Hospital, Huizhou, China.

\section{Xiaoqian Chen}

Department of Pediatrics, The first people's hospital of Foshan, Foshan, China.

\section{Pei Wang}

Department of Pediatrics, The second people's Hospital of Guangdong Province, Guangzhou, China.

\section{Yang Zhou}

GDPH

Jiayi Liang

GDPH

\section{Dian Hong}

GDPH

\section{Zhizhou Shen}

GDPH

\section{Jinjin Yu}

GDPH

\section{Yanhao Wang}

Department of Infectious Diseases, The Ninth People's hospital of DongGuan, Guangzhou, China.

\section{Qiong Meng}

Department of Pediatrics, The second people's Hospital of Guangdong Province, Guangzhou, China.

\section{Yuxin Zhang}

GDPH

\section{Suhua Jiang}

Department of Pediatrics, The first people's hospital of Foshan, Foshan, China. 


\section{Guojun Liu}

Pediatric respiratory department, Dongguan Children's Hospital , Dongguan, China.

\section{Yuxiong Guo (D 2003Kellylaw@163.com)}

Department of Pediatrics, Guangdong Provincial People's Hospital (GDPH)囚Guangdong Academy of Medical Sciences $\llbracket$ Guangzhou, China.

\section{Research Article}

Keywords: SARS-CoV-2, 2019-nCoV, children, COVID-19, new coronavirus infection

Posted Date: April 7th, 2020

DOI: https://doi.org/10.21203/rs.3.rs-21625/v1

License: (c) (i) This work is licensed under a Creative Commons Attribution 4.0 International License.

Read Full License 


\section{Abstract}

Background: The number of coronavirus disease 2019 (COVID-19) cases caused by severe acute respiratory syndrome coronavirus 2 (SARS-CoV-2) infection has significantly increased, and the disease is rapidly spreading to all parts of the country and around the world. A retrospective study of children with SARS-CoV-2 provides a reference for the diagnosis and treatment of children during this epidemic.

Methods: We retrospectively studied 12 cases of children with viral infection caused by SARS-CoV-2 admitted to 6 hospitals in Guangdong Province between January 25, 2020, and February 12, 2020, and analyzed the clinical features and outcomes of the patients.

Results: A total of 12 children with SARS-CoV-2 infection from 6 hospitals were enrolled in the study; 6 were boys. The mean age was $9.8 \pm 4.7$ years, with a minimum age of 2 years and 10 months. The mean body weight was $37.3 \pm 23.6 \mathrm{~kg}$, with a minimum body weight of $13.0 \mathrm{~kg}$. There were no severe cases or critical severe cases. There were 2 cases of mild pneumonia (16.7\%), 7 cases of acute upper respiratory tract infection (58.3\%), and 3 cases of latent infection (25.0\%). In terms of symptoms, there were 7 cases of fever (58.3\%), 5 cases of cough ( $41.7 \%), 3$ cases of runny nose $(25.0 \%), 2$ cases of systemic fatigue and soreness $(16.7 \%)$, and 4 cases of no symptoms (33.3\%). Three patients $(75.0 \%)$ showed decreased white blood cell (WBC) counts for their first complete blood count (CBC) after admission, and one patient (8.3\%) had a low lymphocyte count. There were no obvious abnormalities in C-reactive protein (CRP, 1.53 $\pm 2.28 \mathrm{mg} / \mathrm{l})$, procalcitonin (PCT, $0.21 \pm 0.13 \mathrm{ng} / \mathrm{ml})$, or coagulation function. No abnormalities were detected for creatine kinase (CK), creatine kinase-MB (CKMB), lactate dehydrogenase (LDH), aspartate aminotransferase (AST), alanine aminotransferase (ALT), blood urea nitrogen (BUN) and serum creatinine (Scr). Six cases (50.0\%) were positive for Mycoplasma pneumoniae antibodies. 2 cases showed pulmonary exudative lesions on chest X-ray or computed tomography (CT). All children tested positive for SARS-CoV-2 by real-time reverse-transcription-polymerase-chain-reaction (RT-PCR) assays of throat swabs. 9 patients received antiviral treatment with lopinavir/ritonavir. All patients received symptomatic supportive treatment and were quarantined, and their conditions improved. There was no respiratory failure, acute respiratory distress syndrome, shock complications, or death observed for any case. All patients recovered and were discharged, with an average length of hospital stay of 14 days.

Conclusions: This study with a small sample size suggests that all SARS-CoV-2-infected children had normal or reduced WBCs; however, fever was not as common as expected, and a decrease in lymphocyte count was rare. The clinical manifestations of SARS-CoV-2 infection in children are mild, COVIDQ19 is rare, and the prognosis is good. But the presence of latent SARS-CoV-2 infection in children presents new challenges for effective clinical prevention and control.

\section{Background}


Since December 2019, the number of patients with coronavirus disease 2019 (COVID-19) caused by the severe acute respiratory syndrome coronavirus 2 (SARS-CoV-2) has increased significantly. It quickly spread to other cities and provinces in China as well as other countries and regions in the world. Cases of patients with COVID-19 have emerged in the United States, Canada, Singapore, Europe and other

countries and regions ${ }^{[1-5]}$, and the disease has shown a spreading trend. As of March 30, 2020, more than 500,000 cumulative confirmed cases of COVID-19 caused by SARS-CoV- 2 have been reported all over the world, and more than 20,000 deaths have been reported. SARS-CoV-2 is spread mainly through respiratory droplets and contact, with the characteristics of human-to-human transmission and family cluster outbreak, and the population is generally susceptible ${ }^{[6-8]}$. Children are less likely to be infected with SARS-CoV-2 than adults ${ }^{[9,10]}$. Studies analyzing the clinical characteristics of COVID-19 in children are rare. Therefore, this study collected the clinical data of children with SARS-CoV-2 admitted to 6 hospitals in Guangdong Province for retrospective analysis. This study aims to provide a reference for the diagnosis and treatment of infected children during the SARS-CoV-2 epidemic.

\section{Data And Methods}

\section{Subjects}

A total of 12 children with SARS-CoV-2 aged between 0 and 18 years (excluding newborns) admitted to our 6 hospitals from January 25, 2020, to February 12,2020, were included in this study. These 6 hospitals are Guangdong Provincial People's Hospital, The second people's Hospital of Guangdong Province, The Ninth People's hospital of DongGuan, Huizhou Third People's Hospital and Dongguan Children's Hospital, The first people's hospital of Foshan. The diagnostic criteria, diagnosis and treatment principles, and discharge criteria for children with COVID-19 ${ }^{[9,11]}$ were determined according to the Expert Consensus on the Diagnosis and Treatment of Novel Coronavirus Pneumonia.

\section{Methods}

Identical data collection forms were sent to researchers in the cooperative organization to retrospectively collect the clinical data of children with SARS-CoV-2 infection. The investigations and forms were completed using the patients' medical records. Statistical analysis was conducted by the Guangdong Provincial People's Hospital. Clinical data included age, sex, body weight, epidemiological investigation, clinical signs and symptoms, laboratory and imaging data, major diagnosis and treatment plan, and prognosis. The SARS-CoV-2 test using throat swabs was performed at the local Centers for Disease Control (CDC). All specimens were tested for the SARS-CoV-2 nucleic acid using real-time reversetranscription polymerase chain reaction (RT-PCR). This study was approved by Research Ethics Committee, Guangdong General Hospital, Guangdong Acedemy of Medical Sciences, No.GDREC2020013H(RI). 


\section{Statistical methods}

A retrospective design was used in this study, and IBM SPSS (Statistical Package for the Social Sciences) version 19.0 software (SPSS Inc) was used for the statistical description of the data. All continuous numerical variables are represented as the mean \pm standard deviation $( \pm s)$.

\section{Results}

This retrospective study included a total of 12 children, including 6 boys, with a male to female ratio of 1:1. The mean age was $9.8 \pm 4.7$ years, with the minimum age of 2 years and 10 months and maximum age of 17 years. The mean body weight was $37.3 \pm 23.6 \mathrm{~kg}$, with a minimum body weight of $13 \mathrm{~kg}$ and a maximum of $68 \mathrm{~kg}$. All the children tested positive for SARS-CoV-2 by RT-PCR using throat swabs upon admission. The patients were classified according to the Diagnosis, Treatment, and Prevention of 2019 Novel Coronavirus Infection in Children: Experts' Consensus Statement. ${ }^{[9]}$ There were no severe cases or critical severe cases. Among the 12 children, there were 2 cases $(16.7 \%)$ with pulmonary exudative changes on pulmonary imaging and mild clinical manifestations such as cough (mild pneumonia), 7 cases (58.3\%) with no abnormalities on pulmonary imaging but only mild symptoms of the upper respiratory tract (acute upper respiratory tract infection), and 3 cases (25.0\%) with no abnormalities in pulmonary imaging and no symptoms (asymptomatic infection, i.e., latent infection). All the children were hospitalized for treatment under quarantine. All children were hospitalized and treated in isolation. At present, all children are cured and discharged. The average hospital stay is 14 days.

All the children with SARS-CoV-2 infection had a history of exposure, i.e., they had been exposed to patients with confirmed SARS-CoV-2 infection, and 4 of them had family cluster outbreak. Fever was the main symptom of these patients at the time of treatment. However, among the 12 children, 6 had fever at admission and 1 had fever during hospitalization; thus, $58.3 \%$ of all patients had fever as a symptom. Five of the children had a moderate or mild fever, and 2 had a high fever during the course of the disease. The longest fever duration among the children was 4 days ( 1 case), and the fever duration of the other 6 children was 1-2 days. Five children (41.7\%) did not have a fever at any point during the disease course. The children with fever were sensitive to antipyretics, and there was no case of high fever that was difficult to control (Table 3). Cough, mainly a dry cough, during the course of disease was the second most common symptom among the children. Five children $(41.7 \%)$ had cough over the course of the disease, while the other 7 children (58.3\%) did not present cough over the course of the disease. Three of the children $(25.0 \%)$ had a runny nose over the course of disease, and the remaining 9 children $(75.0 \%)$ did not. In addition, 2 children $(16.7 \%)$ had manifestations of fatigue and soreness. None of the children presented sore throat, shortness of breath, or cyanosis, nor did they have gastrointestinal symptoms such as headache, vomiting, or abdominal pain. Four children (33.3\%) did not show any discomfort during the entire course of the disease, and the main reason for seeking medical service was a clear history of exposure or cluster outbreak and the positive SAS-CoV-2 results by RT-PCR using throat swabs (Table 1). 
After admission, 3 children (25.0\%) had low white blood cell (WBC) counts at their first complete blood count (CBC), and one patient had a relatively low absolute lymphocyte count (8.3\%). The WBC and lymphocyte counts were $5.90 \pm 2.47 \times 10^{9} / \mathrm{L}$ and $2.29 \pm 1.28 \times 10^{9} / \mathrm{L}$, respectively. C-reactive protein (CRP) levels were $(1.56 \pm 2.28 \mathrm{mg} / \mathrm{l})$ for the first laboratory test during hospitalization. One patient was not tested for procalcitonin (PCT) after admission; for the other children, the PCT level was $0.21 \pm 0.13$ $\mathrm{ng} / \mathrm{ml}$. The PCT level in 8 children was above $0.1 \mathrm{ng} / \mathrm{ml}$ but lower than $0.5 \mathrm{ng} / \mathrm{ml}$, suggesting a slight increase. Three children (27.3\%) had a negative PCT. Coagulation tests were not performed for one patient, and the other 11 children had a prothrombin time (PT) of $13.3 \pm 1.5$ seconds, an activated partial thromboplastin time (APTT) of $37.5 \pm 5.8$ seconds, and a fibrinogen (FIB) level of $3.10 \pm 1.14 \mathrm{~g} / \mathrm{L}$. Overall, these three coagulation indicators did not show significant abnormalities; however, the FIB level in one child was abnormally high. The 11 children underwent cardiac enzyme tests during hospitalization. Creatine kinase (CK) was $81.1 \pm 29.2 \mathrm{U} / \mathrm{L}$, creatine kinase-MB (CKMB) was $16.5 \pm 5.5 \mathrm{U} / \mathrm{L}$, lactate dehydrogenase (LDH) was $192.6 \pm 39.5 \mathrm{U} / \mathrm{L}$ ), and alanine aminotransferase (ALT) was $15.9 \pm 10.7 \mathrm{U} / \mathrm{L}$, all within the normal range. The blood urea nitrogen (BUN) and serum creatinine (Scr) levels of 9 children were $4.3 \pm 1.0 \mathrm{mmol} / \mathrm{l}$ and $46.0 \pm 15.4 \mu \mathrm{mol} / \mathrm{l}$, respectively, with no evidence of significant impairment of renal function. Nine children completed chest computed tomography (CT) scans, and pulmonary exudative lesions were found in 1 children (positive rate 12.5\%). Four children completed chest X-ray examinations, and the examination suggested lung exudation in 1 case (positive rate 25.0\%). Of the 12 children, $2(16.7 \%)$ had pulmonary exudative lesions on chest images, but exudative lesions were very slight (Table 2).

During inpatient treatment, nine children (75.0\%) received antiviral therapy with lopinavir/ritonavir tablets, and two children (16.7\%) received interferon inhalation. Three children (25.0\%) required oxygen therapy, which was low-flow or high-flow oxygen inhalation. There was no case of respiratory failure or acute respiratory distress syndrome (ARDS). None of the pediatric patients received glucocorticoid therapy or intravenous gamma globulin. Six children (50.0\%) had positive results for the detection of Mycoplasma pneumoniae, and all received oral azithromycin. After treatment, the children's conditions improved, with no cases of shock or ARDS. During the course of the disease, the children did not require mechanical ventilation, blood purification or extracorporeal membrane oxygenation (ECMO). No complications occurred in the 12 children; the conditions of all children were stable, and they gradually improved. All were cured and discharged (Table 3).

\section{Discussion}

Since December 2019, pneumonia of unknown etiology has emerged in the Wuhan area of Hubei, China. A novel $\beta$-coronavirus was discovered by whole genome sequencing of patients' alveolar lavage fluid ${ }^{[12]}$. World Health Organization (WHO) and related institutions have named it severe acute respiratory syndrome coronavirus 2 (SARS-CoV-2). SARS-CoV-2 might be derived from wild animals, but its specific origins still need further confirmation. Patients with COVID-19 are highly contagious and are the main source of infection. The transmission route is mainly via respiratory droplets and contact ${ }^{[9]}$. 
Gastrointestinal symptoms are common in patients with COVID-19 and the faeces were infectious ${ }^{[13]}$. Currently, there is no sufficient evidence to prove the presence of vertical transmission ${ }^{[14]}$. In terms of the current epidemic situation, there have been few confirmed cases in children ${ }^{[10]}$, and most infected children have had relatively mild symptoms ${ }^{[9]}$. This is similar to the findings of this retrospective study. Although the number of pediatric cases is small, the disease is difficult to diagnose, treat and quarantine, and extra attention should be given ${ }^{[15]}$.

All the pediatric patients in this retrospective study had a clear history of exposure to patients diagnosed with SARS-CoV-2 infection, and some of them had a family cluster outbreak. Dawei Wang et al ${ }^{[3,17]}$ retrospectively analyzed 138 patients with COVID-19 and found that fever and cough were common in adults with COVID-19: $98.6 \%$ had fever, and 59.4\% had cough. However, in children, only $41.7 \%$ had cough during the course of the disease, $58.3 \%$ had fever, and $41.7 \%$ had no fever throughout the course of the disease, which presents new challenges for use of fever and cough for the early screening of children carrying SARS-CoV-2. In addition, up to $33.3 \%$ of the patients were completely asymptomatic throughout the course of the disease. For these children, it was particularly important to undergo screening methods and seek treatment soon as possible after exposure to SARS-CoV-2 infection. Children carrying SARS-CoV-2 without any discomfort may be potential sources of infection. Early identification of children with latent infection of SARS-CoV-2 is a difficulty in clinical practice and poses new challenges for the effective prevention and control of SARS-CoV-2. Lymphocyte reduction occurred in $70.3 \%$ of adults with COVID-19 ${ }^{[16]}$. However, this study showed that only $8.3 \%$ of children with SARSCoV-2 infection had decreased lymphocyte counts. Therefore, the sensitivity lymphocyte counts for the diagnosis of SARS-CoV-2 in children is low. Normal or decreased WBCs, normal CRP, and PCT $<0.5$ were found in all of the children with SARS-CoV-2 infection in this study, which is similar to the presentation of children with common viral infection.

None of the children included in this study were infants; there were 2 patients under 3 years of age and 10 patients over 4 years of age, and the average age was 9.8 years. The reason for the low proportion of infants and young children is not clear, but it may be related to their small range of activities and low probability of contact with people with SARS-CoV-2 infection and to their immune function. No evidence of significant damage was found in coagulatory function or in the liver, kidney, or heart of any of the pediatric patients. The overall clinical manifestations of SARS-CoV-2 infections in children are relatively mild. The proportion of COVID-19 pneumonia cases is small (16.7\%) and the prognosis is good. Currently, some drugs may be effective for COVID-19 treatment ${ }^{[17]}$, but there is not enough evidence to indicate which those are. There is no specific drug for patients with COVID-19 in clinical practice, and quarantine and supportive care for symptoms are the main treatment measures ${ }^{[9,18]}$. In this study, $75.0 \%$ of the children received lopinavir/ritonavir tablets for antiviral treatment, and $25.0 \%$ of the pediatric patients did not use this drug. From clinical observation, No significant difference was found between the two groups of patients, and no obvious drug side effects were observed, possibly due to the small sample size. After active symptomatic support treatment, all of the children's condition gradually improved, and no respiratory failure, ARDS, or shock was observed. None of the children required intensive care unit 
(ICU) admission, and none had serious conditions requiring mechanical ventilation, blood purification, or ECMO supportive treatment. Compared with adults, children with COVID-19 have a milder presentation ${ }^{[6}$, 16]. Unlike severe adult patients with underlying disease such as diabetes, hypertension ${ }^{[16]}$, children with SARS-CoV-2 infections are susceptible to mycoplasma infection. This study found that half of the children were complicated with mycoplasma infection. The reason for this finding is not clear. It may be related to the decline in body immunity caused by the viral infection and the impairment of the airway protection barrier.

The findings of this study can provide a reference for the diagnosis and treatment of children with SARSCoV-2 infections in clinical practice. However, all the cases in this study were from hospitals in Guangdong Province, and the number of cases was relatively small. The clinical manifestations of the disease were generally mild and lack representativeness. To obtain more reliable characteristics of SARSCoV-2 infections in children, it is necessary to further increase the sample size and prolong the observation time.

\section{Conclusion}

In summary, the clinical study of 12 pediatric patients in this study suggested that all children with SARSCoV-2 infection had normal or reduced WBCs, but fever was not as common as expected, the reduction of lymphocyte counts was rare. The clinical manifestations of SARS-CoV-2 infection in children are mild, COVID-19 is rare, and prognosis is good. The history of exposure to COVID-19 is important when screening children for SARS-CoV-2 infection. Nonetheless, the presence of SARS-CoV-2 latent infection in children presents new challenges for effective clinical prevention and control.

\section{Abbreviations}

COVID-19:coronavirus disease 2019; SARS-CoV-2: severe acute respiratory syndrome coronavirus 2; WBC: white blood cell; CRP: C-reactive protein; PCT: procalcitonin; CK: creatine kinase; CKMB: creatine kinase-MB; LDH: lactate dehydrogenase; AST: aspartate aminotransferase; ALT: alanine aminotransferase; BUN: blood urea nitrogen; Scr: serum creatinine; CT: computed tomography.

\section{Declarations}

\section{Ethics approval and consent to participate}

The data used in this study was anonymised before its use. And the study received the written consent of lead unit Guangdong General Hospital, Guangdong Acedemy of Medical Sciences: Research Ethics Committee, No.GDREC2020013H(RI). At the same time, This study received verbal consent from Medical Ethics Committee of Dongguan Children's Hospital (Eighth People's Hospital of Dongguan), Medical Ethics Committee of Huizhou Third People's Hospital, Research Ethics Committeeof The second people's 
Hospital of Guangdong Province, Medical Ethics Committee of The first people's hospital of Foshan, and Institutional Review Board of The Ninth People's hospital of DongGuan.

\section{Consent for publication}

All authors and all author units agree to publish the article.

\section{Availability of data and materials}

We confirm that these patients have not been reported in any other submission by you or anyone else. The datasets supporting the conclusions of this article are included within the article.

\section{Competing interests}

There are no conflicts of interest.

\section{Funding}

The study was funded by Medical Scientific Research Foundation of Guangdong Province of China (grant number A2017066, A2017046), Matching funds for Distinguished Young Medical Talents of "Climbing" program in Guangdong, China (grant number KJ012019451), and Natural Science Foundation of Guangdong Province of China (grant number 2017A030310605).

\section{Authors' contributions}

Guilang $Z$ and Yuxiong $G$ designed the study, Guilang $Z$ wrote the paper. All authors conducted the clinical studies, Chuxing $X$, Guojun $L$ and Yuxiong $G$ analysed the data. All authors contributed to the study implementation, interpretation and reporting. Guilang $Z$ and Chuxing $X$ contributed equally to this work. All authors have read and approved the manuscript.

\section{Acknowledgements}

We would like to express our gratitude to all hospitals (Guangdong Provincial People's Hospital, The second people's Hospital of Guangdong Province, The Ninth People's hospital of DongGuan, Huizhou Third People's Hospital and Dongguan Children's Hospital and The first people's hospital of Foshan) and to all levels of the Centers for Disease Control (CDC) for their strong support. At the same time, I would like to thank the AJE team for their great help in the language of this article. 


\section{References}

1.Cowling BJ, Leung GM: Epidemiological research priorities for public health control of the ongoing global novel coronavirus (2019-nCoV) outbreak. Euro Surveill 2020.

2.Liu J, Liao X, Qian S, Yuan J, Wang F, Liu Y, Wang Z, Wang FS, Liu L, Zhang Z: Community Transmission of Severe Acute Respiratory Syndrome Coronavirus 2, Shenzhen, China, 2020. Emerging infectious diseases 2020, 26(6).

3.Arashiro T, Furukawa K, Nakamura A: COVID-19 in 2 Persons with Mild Upper Respiratory Symptoms on a Cruise Ship, Japan. Emerging infectious diseases 2020, 26(6).

4.Hodcroft EB: Preliminary case report on the SARS-CoV-2 cluster in the UK, France, and Spain. Swiss medical weekly 2020, 150(9-10).

5.Deng CX: The global battle against SARS-CoV-2 and COVID-19. International journal of biological sciences 2020, 16(10):1676-1677.

6.Zhu N, Zhang D, Wang W, Li X, Yang B, Song J, Zhao X, Huang B, Shi W, Lu R et al: A Novel Coronavirus from Patients with Pneumonia in China, 2019. The New England journal of medicine 2020.

7.Huang C, Wang Y, Li X, Ren L, Zhao J, Hu Y, Zhang L, Fan G, Xu J, Gu X et al: Clinical features of patients infected with 2019 novel coronavirus in Wuhan, China. Lancet 2020.

8.Chan JF, Yuan S, Kok KH, To KK, Chu H, Yang J, Xing F, Liu J, Yip CC, Poon RW et al: A familial cluster of pneumonia associated with the 2019 novel coronavirus indicating person-to-person transmission: a study of a family cluster. Lancet 2020.

9.Shen $\mathrm{K}$, Yang Y, Wang T, Zhao D, Jiang Y, Jin R, Zheng Y, Xu B, Xie Z, Lin L et al: Diagnosis, treatment, and prevention of 2019 novel coronavirus infection in children: experts' consensus statement. World journal of pediatrics: WJP 2020.

10.Gan H, Zhang Y, Yuan M, Wu XY, Liu ZR, Liu M, Wu JB, Xu SJ, Gong L, Xu HL et al: [Epidemiological analysis on 1052 cases of COVID-19 in epidemic clusters]. Zhonghua liu xing bing xue za zhi = Zhonghua liuxingbingxue zazhi 2020, 41(5):E027.

11.Chen ZM, Fu JF, Shu Q, Chen YH, Hua CZ, Li FB, Lin R, Tang LF, Wang TL, Wang W et al: Diagnosis and treatment recommendations for pediatric respiratory infection caused by the 2019 novel coronavirus. World journal of pediatrics: WJP 2020.

12.Lu R, Zhao X, Li J, Niu P, Yang B, Wu H, Wang W, Song H, Huang B, Zhu N et al: Genomic characterisation and epidemiology of 2019 novel coronavirus: implications for virus origins and receptor binding. Lancet 2020. 
13.Tian Y, Rong L: Review article: Gastrointestinal features in COVID-19 and the possibility of faecal transmission. 2020.

14.Zhu H, Wang L, Fang C, Peng S, Zhang L, Chang G, Xia S, Zhou W: Clinical analysis of 10 neonates born to mothers with 2019-nCoV pneumonia. Translational pediatrics 2020, 9(1):51-60.

15.Li Y, Guo F: Insight into COVID-2019 for pediatricians. 2020.

16.Wang D, Hu B, Hu C, Zhu F, Liu X, Zhang J, Wang B, Xiang H, Cheng Z, Xiong Y et al: Clinical Characteristics of 138 Hospitalized Patients With 2019 Novel Coronavirus-Infected Pneumonia in Wuhan, China. JAMA: the journal of the American Medical Association 2020.

17.Elfiky AA: Ribavirin, Remdesivir, Sofosbuvir, Galidesivir, and Tenofovir against SARS-CoV-2 RNA dependent RNA polymerase (RdRp): A molecular docking study. Life Sci 2020:117592.

18.Cao B, Wang Y, Wen D, Liu W, Wang J, Fan G, Ruan L, Song B, Cai Y, Wei M et al: A Trial of LopinavirRitonavir in Adults Hospitalized with Severe Covid-19. The New England journal of medicine 2020.

\section{Tables}

Table 1. Clinical manifestations of children with SARS-CoV-2 infection at admission

\begin{tabular}{ccccccccccccc}
\hline Case No. & 1 & 2 & 3 & 4 & 5 & 6 & 7 & 8 & 9 & 10 & 11 & 12 \\
\hline Epidemiological history & + & + & + & & & & & & & & & \\
Fever & + & - & + & + & - & - & + & + & + & + & - & - \\
Pharyngeal pain & - & - & - & - & - & - & - & - & - & - & - & - \\
Runny nose & - & - & + & - & - & - & - & - & + & + & - & - \\
Dry cough & + & + & + & - & - & - & - & - & + & + & - & - \\
Dyspnoea & - & - & - & - & - & - & - & - & - & - & - & - \\
Cyanosis & - & - & - & - & & - & - & - & - & - & - & - \\
Fatigue & + & - & - & - & - & - & - & + & - & - & - & - \\
Headache & - & - & - & - & - & - & - & - & - & - & - & - \\
Gastrointestinal symptoms & - & - & - & - & - & - & - & - & - & - & - & - \\
Other discomfort & - & - & - & - & - & - & - & - & - & - & - & - \\
\hline
\end{tabular}

Table 2. Results of the first laboratory examination and imaging of children with SARSCoV-2 infection at admission 


\begin{tabular}{|c|c|c|c|c|c|c|c|c|c|c|c|c|}
\hline Case No. & 1 & 2 & 3 & 4 & 5 & 6 & 7 & 8 & 9 & 10 & 11 & 12 \\
\hline WBC,$\times 10^{9}$ & 3.37 & 3.97 & 6.83 & 3.03 & 4.23 & 3.74 & 8.42 & 3.19 & 8.49 & 8.23 & 8.24 & 9.00 \\
\hline Lym, $\times 10^{9}$ & 1.46 & 1.25 & 4.31 & 1.41 & 1.82 & 1.47 & 2.85 & 1.79 & 2.44 & 0.61 & 3.25 & 4.80 \\
\hline $\mathrm{CRP}, \mathrm{mg} / \mathrm{l}$ & 1.30 & 0.50 & 8.21 & 0.50 & 0.50 & 0.50 & 1.76 & 3.40 & 1.00 & 1.00 & $<0.05$ & $<0.05$ \\
\hline PCT,ng/ml & 0.21 & 0.18 & 0.28 & 0.42 & 0.22 & 0.21 & 0.16 & $<0.05$ & I & 0.44 & $<0.05$ & $<0.05$ \\
\hline SARS-CoV-2 PCR & + & + & + & + & + & + & + & + & + & + & + & + \\
\hline PT, S & 12.7 & 15.3 & 12.5 & 12.8 & I & 14.1 & 13.4 & 14.3 & 14.8 & 14.4 & 10.5 & 11.1 \\
\hline FIB, $g / 1$ & 3.28 & 2.72 & 2.79 & 6.04 & I & 3.81 & 3.10 & 2.98 & 2.55 & 3.14 & 1.91 & 1.74 \\
\hline APTT, S & 34.1 & 36.3 & 38.8 & 34.5 & l & 39.2 & 34.1 & 49.8 & 41.9 & 42.8 & 29.5 & 31.3 \\
\hline $\mathrm{CK}, \mathrm{U} / \mathrm{L}$ & 60 & 83 & 114 & 72 & 67 & 72 & 72 & 45 & 138 & 116 & 53 & I \\
\hline $\mathrm{CKMB}, \mathrm{U} / \mathrm{L}$ & 9.9 & 14.1 & 24.2 & 8.3 & 24.4 & 12.9 & 22.7 & 17.0 & 14.0 & 18.7 & 16.0 & I \\
\hline LDH, U/L & 169 & 127 & 222 & 198 & 186 & 143 & 222 & 165 & 226 & 260 & 200 & I \\
\hline AST, U/L & 14.2 & 12.9 & 35.0 & 50.0 & 17.0 & 13.3 & 25.3 & 15.0 & 20.0 & 37.0 & 19.0 & I \\
\hline ALT, U/L & 10.2 & 11.8 & 13.1 & 24.3 & 9.3 & 8.2 & 45.4 & 15.0 & 12.0 & 16.0 & 10.0 & I \\
\hline Scr, umol/l & 49 & 48 & 26 & 38 & 43 & 70 & 59 & l & 58 & 23 & I & I \\
\hline BUN, mmol/1 & 2.5 & 3.6 & 3.8 & 5.3 & 4.9 & 4.2 & 3.5 & I & 5.4 & 5.3 & I & I \\
\hline Chest X-ray & I & I & + & I & I & I & I & - & - & - & I & I \\
\hline Chest CT & - & + & I & - & - & - & - & I & - & - & - & - \\
\hline
\end{tabular}

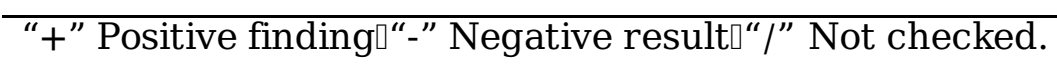

Table 3. Diagnosis and prognosis of children with SARS-CoV-2 infection

\begin{tabular}{|c|c|c|c|c|c|c|c|c|c|c|c|c|}
\hline Case No. & 1 & 2 & 3 & 4 & 5 & 6 & 7 & 8 & 9 & 10 & 11 & 12 \\
\hline Lopinavir/litonavir & + & + & + & + & + & + & + & - & + & + & - & - \\
\hline interferon- $\alpha$ & - & - & + & - & + & - & - & - & - & - & - & - \\
\hline Glucocorticoids & - & - & - & - & - & - & - & - & - & - & - & - \\
\hline Oxygen therapy & + & - & + & - & - & + & - & - & - & - & - & - \\
\hline Fever duration, $\mathrm{d}$ & 2 & 0 & 2 & 2 & 0 & 0 & 2 & 1 & 2 & 4 & 0 & 0 \\
\hline Mycoplasma infection & - & - & - & + & + & + & + & - & + & + & + & - \\
\hline Complication & - & - & - & - & - & - & - & - & - & - & - & - \\
\hline Prognosis & Dis & Dis & Dis & Dis & Dis & Dis & Dis & Dis & Dis & Dis & Dis & Dis \\
\hline Hospital stay, d & 12 & 18 & 19 & 19 & 20 & 11 & 11 & 14 & 5 & 5 & 15 & 15 \\
\hline
\end{tabular}

